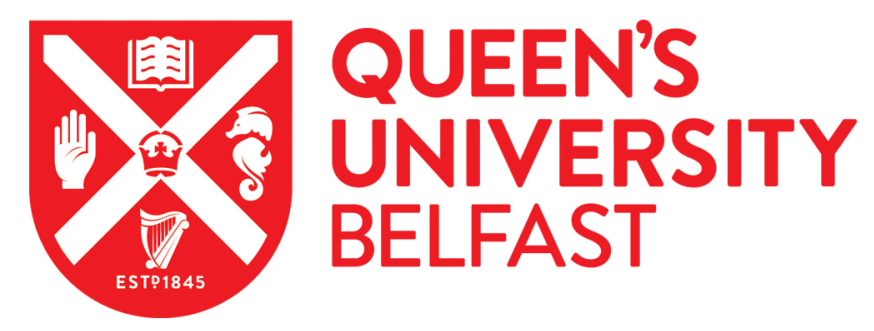

\title{
Epitaxial strain and electric boundary condition effects on the structural and ferroelectric properties of $\mathrm{BiFeO} 3$ films
}

Johann, F., Morelli, A., Biggemann, D., Arredondo-Arechavala, M., \& Vrejoiu, I. (2011). Epitaxial strain and electric boundary condition effects on the structural and ferroelectric properties of BiFeO3 films. Physical Review $B$ (Condensed Matter), 84(9), [094105]. https://doi.org/10.1103/PhysRevB.84.094105

Published in:

Physical Review B (Condensed Matter)

Document Version:

Publisher's PDF, also known as Version of record

Queen's University Belfast - Research Portal:

Link to publication record in Queen's University Belfast Research Portal

Publisher rights

(c) 2011 American Physical Society

\section{General rights}

Copyright for the publications made accessible via the Queen's University Belfast Research Portal is retained by the author(s) and / or other copyright owners and it is a condition of accessing these publications that users recognise and abide by the legal requirements associated with these rights.

Take down policy

The Research Portal is Queen's institutional repository that provides access to Queen's research output. Every effort has been made to ensure that content in the Research Portal does not infringe any person's rights, or applicable UK laws. If you discover content in the Research Portal that you believe breaches copyright or violates any law, please contact openaccess@qub.ac.uk. 


\title{
Epitaxial strain and electric boundary condition effects on the structural and ferroelectric properties of $\mathrm{BiFeO}_{3}$ films
}

\author{
Florian Johann," Alessio Morelli, Daniel Biggemann, Miryam Arredondo, and Ionela Vrejoiu \\ Max Planck Institute of Microstructure Physics, Weinberg 2, D-06120 Halle, Germany \\ (Received 17 May 2011; revised manuscript received 22 August 2011; published 19 September 2011)
}

\begin{abstract}
The influence of both compressive and tensile epitaxial strain along with the electrical boundary conditions on the ferroelastic and ferroelectric domain patterns of bismuth ferrite films was studied. $\mathrm{BiFeO}_{3}$ films were grown on $\mathrm{SrTiO}_{3}(001), \mathrm{DyScO}_{3}(110), \mathrm{GdScO}_{3}(110)$, and $\mathrm{SmScO}_{3}(110)$ substrates to investigate the effect of room temperature in-plane strain ranging from $-1.4 \%$ to $+0.75 \%$. Piezoresponse force microscopy, transmission electron microscopy, $\mathrm{x}$-ray diffraction measurements, and ferroelectric polarization measurements were performed to study the properties of the films. We show that $\mathrm{BiFeO}_{3}$ films with and without $\mathrm{SrRuO}_{3}$ bottom electrode have different growth mechanisms and that in both cases reduction of the domain variants is possible. Without $\mathrm{SrRuO}_{3}$, stripe domains with reduced variants are formed on all rare earth scandate substrates because of their monoclinic symmetry. In addition, tensile strained films exhibit a rotation of the unit cell with increasing film thickness. On the other side, the presence of $\mathrm{SrRuO}_{3}$ promotes step flow growth of $\mathrm{BiFeO}_{3}$. In case of vicinal $\mathrm{SrTiO}_{3}$ and $\mathrm{DyScO}_{3}$ substrates with high quality $\mathrm{SrRuO}_{3}$ bottom electrode and a low miscut angle of $\approx 0.15^{\circ}$ we observed suppression of the formation of certain domain variants. The quite large in-plane misfit of $\mathrm{SrRuO}_{3}$ with $\mathrm{GdScO}_{3}$ and $\mathrm{SmScO}_{3}$ prevents the growth of high quality $\mathrm{SrRuO}_{3}$ films and subsequent domain variants reduction in $\mathrm{BiFeO}_{3}$ on these substrates, when $\mathrm{SrRuO}_{3}$ is used as a bottom electrode.
\end{abstract}

DOI: 10.1103/PhysRevB.84.094105

PACS number(s): 77.80.Dj, 68.37.Yz, 68.55.-a, 81.15.Aa

\section{INTRODUCTION}

Multiferroic materials stimulated a great deal of investigations because of their potential coupling between electric and magnetic order parameters, making them interesting for both fundamental research and applications. ${ }^{1,2}$ Bismuth ferrite $\left(\mathrm{BiFeO}_{3}\right)$ is a very promising multiferroic, being ferroelectric and antiferromagnetic at room temperature. ${ }^{3} \mathrm{BiFeO}_{3}$ is a rhombohedrally distorted perovskite with a room temperature pseudocubic lattice constant $a_{c}=3.96 \AA$ and a distortion angle $\alpha=0.6^{\circ} .^{4}$ It has a high ferroelectric polarization of $\approx 100 \mu \mathrm{C} / \mathrm{cm}^{2}$ along the polar [111] direction. For $(001)_{c^{-}}$ oriented epitaxial $\mathrm{BiFeO}_{3}$ films eight possible ferroelectric variants can occur, corresponding to four different ferroelastic variants, as depicted in Fig. 1 . Streiffer et al. ${ }^{5}$ predicted that rhombohedral ferroelectric films may form complex stripe domain patterns with both $\{100\}$ and $\{101\}$ twin boundaries as a result of this numerousness of polarization directions. In consequence, it is complicated to control the occurrence of particular domain variants. ${ }^{6}$ Furthermore, the existence of many domain variants can suppress the ferroelectric properties of $\mathrm{BiFeO}_{3}{ }^{7}$ Several publications reported on selection of certain domain variants by reducing the substrate symmetry, either by introduction of a high miscut angle on cubic $\mathrm{SrTiO}_{3}(001)^{8}$ or by the use of orthorhombic (110)-cut rare earth scandate substrates. ${ }^{9}$ The rare earth scandates have a $\mathrm{GdFeO}_{3}$-like structure, ${ }^{10}$ where in one orthorhombic unit cell four monoclinic distorted perovskite pseudocubes are merged [Fig. 1(c)]. For (110) $)_{o}$ oriented substrates, two slightly different pseudocubic in-plane parameters occur, which are listed in Table I together with the monoclinic distortion angle $\beta$, for $\mathrm{DyScO}_{3}, \mathrm{GdScO}_{3}$, and $\mathrm{SmScO}_{3}$. As sketched in Fig. 1(c), for these kind of substrates we make the assignment of the orthorhombic $[001]_{o}$ axis as pseudocubic $[100]_{c}$ axis and the $[1 \overline{1} 0]_{o}$ axis as $[010]_{c}$, where $o$ and $c$ stand for the orthorhombic and cubic notation, respectively.
Chu et al. ${ }^{12}$ reported the formation of quasi-onedimensional stripe domain patterns in $\mathrm{BiFeO}_{3}$ films deposited on $\mathrm{DyScO}_{3}(110)$, promoted by careful control of the thickness and growth mode of the $\mathrm{SrRuO}_{3}$ bottom electrode. The same kind of stripe domain pattern was, however, seen for $\mathrm{BiFeO}_{3}$ films grown directly on $\mathrm{DyScO}_{3}(110)$ and $\mathrm{TbScO}_{3}(110)$, and was attributed to the two different in-plane lattice constants of the orthorhombic substrates. ${ }^{9,13,14}$ Recently tensile strained $\mathrm{BiFeO}_{3}$ has been devoted a great deal of theoretical investigations, ${ }^{15}$ however there are not many experimental studies to provide data to be compared with the theoretical predictions. In particular there are no reports about tensile strained $\mathrm{BiFeO}_{3}$ films investigating the domain patterns.

We report on the structural properties, domain patterns, and ferroelectric properties of epitaxial $\mathrm{BiFeO}_{3}$ films grown by pulsed laser deposition on $\mathrm{SrTiO}_{3}(001), \mathrm{DyScO}_{3}(110)$, $\mathrm{GdScO}_{3}(110)$, and $\mathrm{SmScO}_{3}(110)$. With these substrates the effect of an in-plane epitaxial strain ranging from $-1.4 \%$ to $+0.75 \%$ on $\mathrm{BiFeO}_{3}$ films could be investigated.

We found that the symmetry of the substrate and the electrical properties of the bottom interface have a more evident influence on the domain variants than the magnitude and sign of the epitaxial strain. For $\mathrm{BiFeO}_{3}$ films grown directly on the substrate, we observed that: (i) Under tensile stress, $\mathrm{BiFeO}_{3}$ grown on the rare earth scandates exhibit stripe domains with reduced structural variants as reported for compressive stress, ${ }^{9,13}$ but with much finer stripe widths, down to $10 \mathrm{~nm}$. Our investigations suggest that the origin of the variant selection is rather the monoclinic nature of the substrate than the two different in-plane lattice constants, or at least a combination of both. (ii) An additional effect of tensile strain is that, with increasing thickness, the films show a slight rotation of the pseudocubic unit cell.

The situation is different when a $\mathrm{SrRuO}_{3}$ bottom electrode was employed, because of its additional structural and electrical properties: (i) $\mathrm{BiFeO}_{3}$ films on $\mathrm{SrRuO}_{3} / \mathrm{DyScO}_{3}(110)_{o}$ and 
(a)

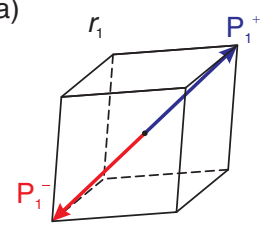

$[001]_{C}$

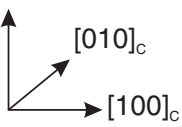

(b)

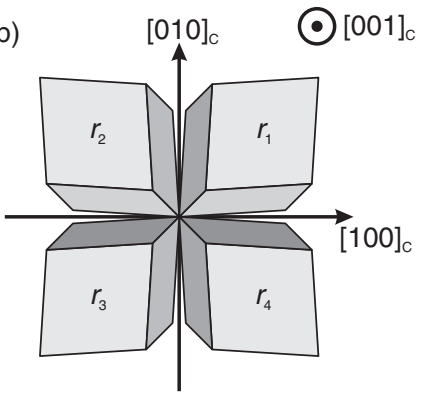

(c) $\begin{array}{ll}{[110]_{\circ}} \\ {[001]_{\mathrm{C}}}\end{array}$

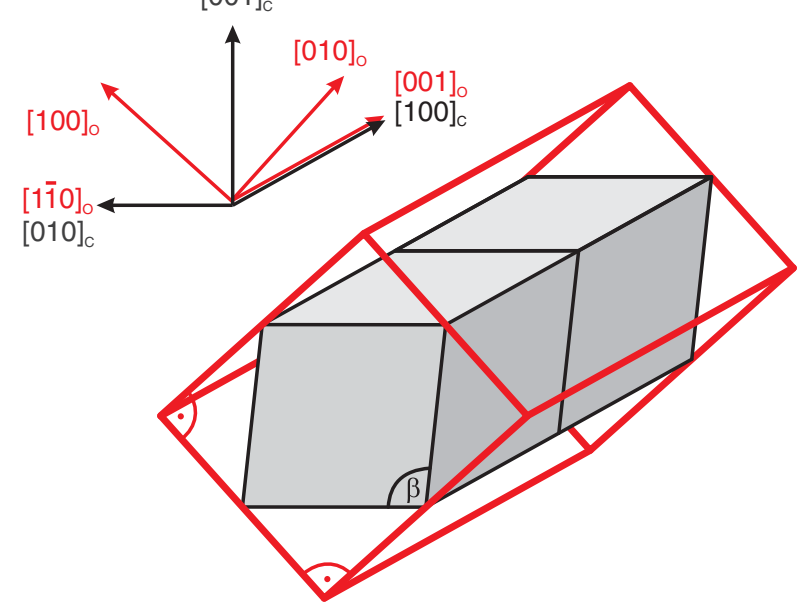

FIG. 1. (Color online) (a) The $\mathrm{BiFeO}_{3}$ perovskite cube is distorted along the direction of polarization. For one distortion two directions for the ferroelectric polarization are possible, shown for variant $r_{1}$. (b) All possible structural variants $r_{1}, r_{2}, r_{3}$, and $r_{4}$, viewed from the top of an epitaxial $(001)_{c}$-orientated $\mathrm{BiFeO}_{3}$ film. (c) Structure of the rare earth scandates with the orthorhombic unit cell in red and two of the four pseudocubic perovskite cells inside the orthorhombic unit cell in black. The pseudocubes are monoclinic distorted with an angle $\beta$.

as well on low miscut $\mathrm{SrRuO}_{3} / \mathrm{SrTiO}_{3}(001)_{c}$ substrates exhibit a reduced number of domain variants due to the electrical properties of $\mathrm{SrRuO}_{3}$ and the miscut direction of the substrate. (ii) In case of $\mathrm{GdScO}_{3}$ and $\mathrm{SmScO}_{3}$ the lattice mismatch of $\mathrm{SrRuO}_{3}$ with these substrates is very high, resulting in a worse film quality and partial strain relaxation. Hence, for

TABLE I. (Pseudo)cubic in-plane lattice constants $a_{c}$ and $b_{c}$ along $\left([010]_{c} \|[110]_{o}\right)$ and $\left([100]_{c} \|[001]_{o}\right)$, respectively, as well as the monoclinic angle $\beta$ of the used substrates and (averaged) in-plane lattice misfit with $\mathrm{BiFeO}_{3}$ and $\mathrm{SrRuO}_{3}$. Values for rare earth scandate lattice constants were taken from Ref. 11.

\begin{tabular}{lccccc}
\hline \hline & & & & \multicolumn{2}{c}{ Misfit with } \\
\cline { 5 - 6 } & $a_{c}(\AA)$ & $b_{c}(\AA)$ & $\beta(\mathrm{deg})$ & $\mathrm{BiFeO}_{3}$ & $\mathrm{SrRuO}_{3}$ \\
\hline $\mathrm{SrTiO}_{3}(001)_{c}$ & 3.905 & 3.905 & & $-1.4 \%$ & $-0.6 \%$ \\
$\mathrm{DyScO}_{3}(110)_{o}$ & 3.946 & 3.952 & 92.8 & $-0.3 \%$ & $+0.5 \%$ \\
$\mathrm{GdScO}_{3}(110)_{o}$ & 3.970 & 3.966 & 92.7 & $+0.2 \%$ & $+1.0 \%$ \\
$\mathrm{SmScO}_{3}(110)_{o}$ & 3.991 & 3.983 & 92.3 & $+0.7 \%$ & $+1.4 \%$ \\
\hline \hline
\end{tabular}
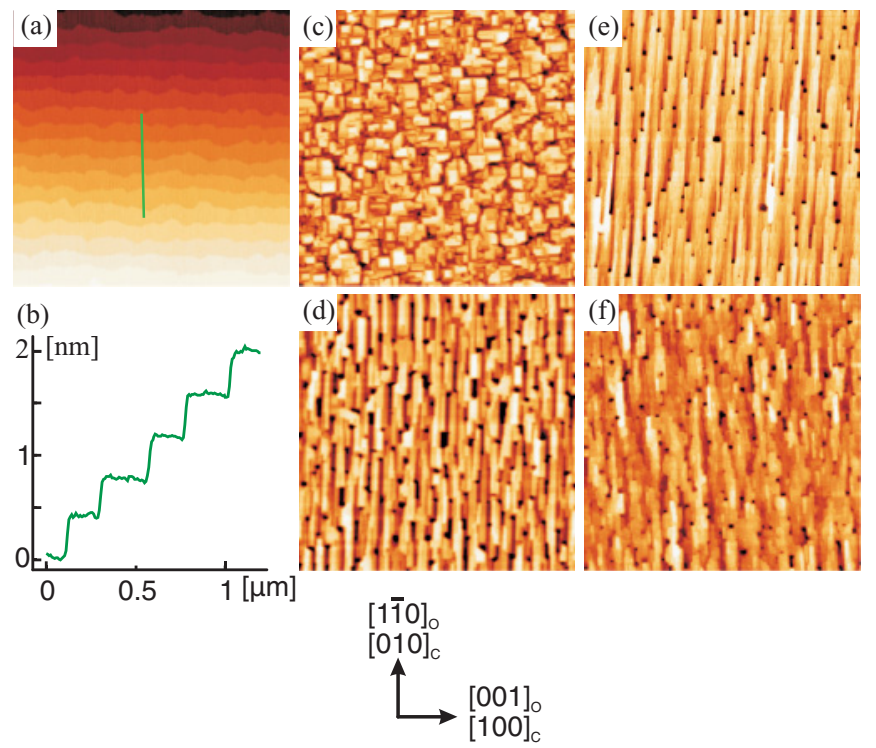

FIG. 2. (Color online) AFM topography of (a) $\mathrm{GdScO}_{3}(110)$ substrate with corresponding scan line in (b). AFM surface morphology of $26 \mathrm{~nm}$ thick $\mathrm{BiFeO}_{3}$ films grown on (c) $\mathrm{SrTiO}_{3}(001)$ (scale: $9 \mathrm{~nm}, \mathrm{rms}: 2.0 \mathrm{~nm}$ ), (d) $\mathrm{DyScO}_{3}(110)$ (scale: $11 \mathrm{~nm}, \mathrm{rms}: 2.7 \mathrm{~nm}$ ), (e) $\mathrm{GdScO}_{3}(110)$ (scale: $4 \mathrm{~nm}$, rms: $1.0 \mathrm{~nm}$ ), and (f) $\mathrm{SmScO}_{3}(110)$ (scale: $7 \mathrm{~nm}$, rms: $1.7 \mathrm{~nm}$ ). All images are $4 \times 4 \mu \mathrm{m}^{2}$.

$\mathrm{BiFeO}_{3}$ films grown on either $\mathrm{GdScO}_{3}$ or $\mathrm{SmScO}_{3}$ with bottom $\mathrm{SrRuO}_{3}$ electrodes, no preferred domain variants could be observed.

Overall, our investigations show that the properties of $\mathrm{BiFeO}_{3}$ films are very sensitive to growth mode, the type of bottom interface, the substrate symmetry and epitaxial strain conditions.

\section{EXPERIMENT}

$\mathrm{BiFeO}_{3}$ films were grown by pulsed laser deposition on $\mathrm{SrTiO}_{3}(001)_{c}, \mathrm{DyScO}_{3}(110)_{o}, \mathrm{GdScO}_{3}(110)_{o}$, and $\mathrm{SmScO}_{3}(110)_{o}$ with and without $\mathrm{SrRuO}_{3}$ bottom electrode. All substrates had a miscut of $0.1^{\circ}$ to $0.15^{\circ}$ toward $[010]_{c}$. $\mathrm{SrTiO}_{3}$ was prepared by an etching and annealing step as reported in Ref. 16, whereas $\mathrm{DyScO}_{3}, \mathrm{GdScO}_{3}$, and $\mathrm{SmScO}_{3}$ were just annealed for $2 \mathrm{~h}$ at 975,1100 , and $1200^{\circ} \mathrm{C}$, respectively. All substrates had uniform, atomically flat terraces of $\approx 200 \mathrm{~nm}$ in width and step heights of one unit cell after preparation. After optimizing the growth conditions, $\mathrm{SrRuO}_{3}$ and $\mathrm{BiFeO}_{3}$ films were deposited at $650{ }^{\circ} \mathrm{C}$ in an $\mathrm{O}_{2}$ pressure of 0.14 mbar. The $\mathrm{BiFeO}_{3}$ films were ablated from stoichiometric targets with a laser fluence of around $0.5 \mathrm{~J} / \mathrm{cm}^{2}$ and a laser repetition rate of $5 \mathrm{~Hz}$, which resulted in a growth rate of $\approx 1 \mathrm{~nm} / \mathrm{min}$. The deposited films were cooled down to room temperature in 200 mbar partial pressure of oxygen with $900^{\circ} \mathrm{C} / \mathrm{h} . \mathrm{BiFeO}_{3}$ films of about 13,26 , and $38 \mathrm{~nm}$ in thickness were deposited directly on the substrates and about $30 \mathrm{~nm}$ thick $\mathrm{BiFeO}_{3}$ films were grown on substrates with $\mathrm{SrRuO}_{3}$ bottom electrode. For this thickness the $\mathrm{BiFeO}_{3}$ film is still fully strained. ${ }^{17} \mathrm{We}$ varied the $\mathrm{SrRuO}_{3}$ thickness from 5 to $50 \mathrm{~nm}$, depending on the extent of misfit of $\mathrm{SrRuO}_{3}$ with the substrate. 
The morphology of the substrates and films were measured with a commercial atomic force microscope (Veeco Digital Instruments D5000) in tapping mode. Piezoresponse force microscopy (PFM) was performed on a XE-100 Park System in contact mode to study the ferroelectric domains and the piezoelectric response of the films. Cantilever with spring constants of $2.8-7.5 \mathrm{~N} / \mathrm{m}$ and tips coated with a conductive layer of $\mathrm{Pt} / \mathrm{Ti}$ or $\mathrm{Pt} / \mathrm{Ir}$ were used. In order to achieve 3D reconstruction of the polarization patterns vertical PFM (VPFM) and lateral PFM (LPFM) images were acquired with two different cantilever orientations. ${ }^{18}$ Plan-view and cross-section specimens for transmission electron microscopy (TEM) characterization were prepared by conventional techniques. ${ }^{19}$ Conventional TEM was performed on both type of samples on a Philips CM20T microscope at $200 \mathrm{kV}$. High resolution TEM (HRTEM) images were obtained from cross-section samples on a Jeol 4010 microscope with $\mathrm{LaB}_{6}$ electron gun, point resolution of $1.6 \AA$ at $400 \mathrm{kV}$. X-ray diffraction (XRD) measurements were done with a Philips X'Pert system. The different structural variants of the $\mathrm{BiFeO}_{3}$ films were studied with XRD reciprocal space maps (RSMs). ${ }^{20,21}$

\section{RESULTS AND DISCUSSION}

Epitaxial $\mathrm{BiFeO}_{3}$ films grown directly on the substrate and those grown on a $\mathrm{SrRuO}_{3}$ bottom electrode layer show different properties. Most evident is the different growth mode of $\mathrm{BiFeO}_{3}$ in the two cases as seen by AFM topography. If the $\mathrm{BiFeO}_{3}$ film is grown directly on the substrate a $3 \mathrm{D}$ growth is observed (Fig. 2), whereas for $\mathrm{SrRuO}_{3}$ bottom electrode the $\mathrm{BiFeO}_{3}$ stays atomically flat (Fig. 8). The origin of this could be the different surface diffusivity of $\mathrm{BiFeO}_{3}$ on the different surface terminations, which is in case of the substrates a B-site termination ${ }^{16,22}$ and in case of $\mathrm{SrRuO}_{3}$ step-flow-grown layers an A-site termination. ${ }^{23}$ Due to these major differences the two cases will be discussed separately, first the $\mathrm{BiFeO}_{3}$ films grown directly on the substrates, followed by the $\mathrm{BiFeO}_{3}$ films with $\mathrm{SrRuO}_{3}$ bottom electrode.

\section{A. $\mathrm{BiFeO}_{3}$ films grown directly on the substrate}

As an example of a prepared substrate morphology, Fig. 2(a) shows the topography of a vicinal $\mathrm{GdScO}_{3}(110)$ substrate and Fig. 2(b) shows its scan line across the terraces. For all films shown in Fig. 2 the orientation of the substrate terraces is as shown in Fig. 2(a). The morphology of the $\mathrm{BiFeO}_{3}$ films grown without $\mathrm{SrRuO}_{3}$ bottom electrode show square island in case of $\mathrm{SrTiO}_{3}$ substrate [Fig. 2(c)] and elongated islands or stripes in case of rare earth scandates [Figs. 2(d)-2(f)]. The elongation is for all films on the three types of rare earth scandates aligned along the $[1 \overline{1} 0]_{o}$ direction, which is the direction of higher in-plane misfit for $\mathrm{BiFeO}_{3}$ with these substrates.

To investigate the ferroelectric domain structure, PFM measurements were performed on all samples, shown in Fig. 3. Measurements were carried out with two cantilever orientations: (A) aligned along $[010]_{c}$ and (B) aligned along $[100]_{c}$. In the VPFM images a dark (bright) contrast corresponds to a polarization pointing outward (inward) the sample surface, while for the LPFM images a black (white) contrast corresponds to a projection of the polarization pointing in the sample plane to the right (left) side of the cantilever. As a result, for both LPFM contrasts two possible directions of polarization are possible, as depicted in the insets of the LPFM images. For all of the films the PFM measurements reveal that the surface morphology is related, to a certain extent, to the ferroelectric/ferroelastic domain patterns. The domain walls almost always match with topographic features for $\mathrm{BiFeO}_{3}$ grown without $\mathrm{SrRuO}_{3}$ bottom electrode.

For films deposited on $\mathrm{SrTiO}_{3}$ the PFM images show similar patterns for both cantilever orientations, therefore only one orientation is shown here [Fig. 3(a)]. The VPFM images reveal that almost the complete film has a polarization pointing upward, with only some stripes pointing downward. The LPFM shows an evenly distributed contrast with domain boundaries matching the island borders seen in the topography image, suggesting that each island is monodomain. Furthermore, the bright stripes seen in the vertical PFM image are positioned at the domain walls seen in the lateral PFM. Combining both VPFM and LPFM images it can be concluded that mostly the four polarization directions pointing upward $\left(P_{1}^{+}, P_{2}^{+}, P_{3}^{+}\right.$, and $P_{4}^{+}$) occur with equal distribution. From this it follows that all four structural variants, $r_{1}$ to $r_{4}$, occur in the $\mathrm{BiFeO}_{3}$ film on $\mathrm{SrTiO}_{3}(001)$ equally.

Investigations of the films deposited on the orthorhombic rare earth scandates show different domain configuration than in case of films deposited on cubic $\mathrm{SrTiO}_{3}$. PFM measurements reveal that the same kind of stripe domains with reduced domain variants appear for both compressive and tensile strain [Figs. 3(b)-3(d)], as reported for compressive strain. ${ }^{9,13}$ For the cantilever alignment (B), exactly the same stripe domain pattern is seen in both VPFM and LPFM images. Hence, it can be concluded that from the four structural variants [see Fig. 1(b)] only two occur, namely $r_{3}$ and $r_{4}$. In case of a cantilever orientation (A) the VPFM domain pattern is similar, but the LPFM domain pattern changes to bigger domain sizes. Within such a domain, the stripes seen in VPFM have an alternating polarization of either $P_{3}^{+}$and $P_{4}^{-}$or $P_{3}^{-}$and $P_{4}^{+}$, both having $109^{\circ}$ domain walls as sketched in Fig. 3(e). The domain walls seen in the LPFM image (A) are either $71^{\circ}$, if there is no corresponding domain wall visible in VPFM, or $180^{\circ}$ domain walls otherwise.

On all three orthorhombic substrates the $\mathrm{BiFeO}_{3}$ stripes with polarization pointing outward appear to be wider than the stripes pointing inward, presumably due to electric interactions at the interface with the highly insulating substrates. The stripe periodicity is $\approx 140 \mathrm{~nm}$ in case of a $\mathrm{DyScO}_{3}$ substrate, whereas on $\mathrm{GdScO}_{3}$ and $\mathrm{SmScO}_{3}$ substrates the stripes are much finer, some of them even with a width of less than $10 \mathrm{~nm}$, which is the limit of lateral resolution for PFM. ${ }^{24}$ As a result the PFM signal is very low and the domain shape can not be resolved in detail.

To circumvent the resolution limit of PFM we employed TEM to investigate the shape and size of the domains of $\mathrm{BiFeO}_{3}$ films subjected to tensile epitaxial strain in more detail. Regarding TEM one is restricted to the investigation of different structural variants (the two different ferroelectric variants of one structural variant can not be visualized), but the lateral resolution is orders of magnitude higher compared to PFM. A plan-view specimen was prepared from a $26 \mathrm{~nm}$ thick $\mathrm{BiFeO}_{3}$ film and a cross section specimen was prepared from 

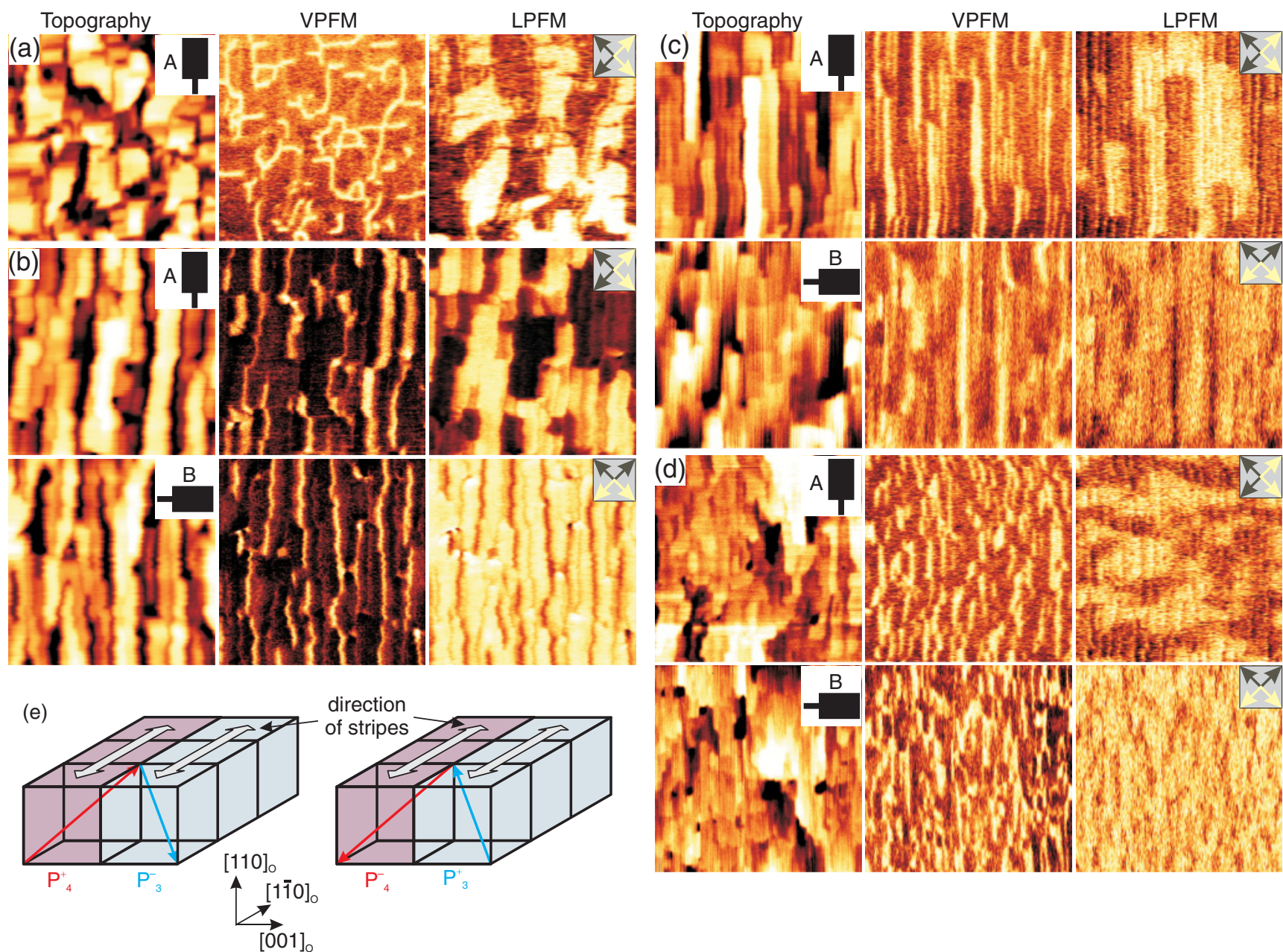

FIG. 3. (Color online) Topography, VPFM and LPFM images (left to right) of $26 \mathrm{~nm}$ thick $\mathrm{BiFeO}_{3}$ films without $\mathrm{SrRuO}_{3}$ bottom electrode on (a) $\mathrm{SrTiO}_{3}$, (b) $\mathrm{DyScO}_{3}$, (c) $\mathrm{GdScO}_{3}$, and (d) $\mathrm{SmScO}_{3}$. Samples were measured with the cantilever aligned along (A) [010] $]_{c}$ and (B) [100] , as depicted in the inset in each topography image. The insets in the LPFM images show the two possible polarization directions for both contrasts. All images are $1 \times 1 \mu \mathrm{m}^{2}$. (e) Schematic representation of domain configuration with $109^{\circ}$ domain boundaries for $\mathrm{BiFeO}_{3}$ films grown on rare earth scandates.

the $38 \mathrm{~nm}$ thick $\mathrm{BiFeO}_{3}$, both grown on $\mathrm{SmScO}_{3}(110)$. As can be seen in Fig. 4(a), the stripe domains observed by plan-view TEM match in size with those seen by PFM. Most of the stripe domains end in a needle-like shape and merge alternating with $\pm 45^{\circ}$ with respect to the stripe direction. The averaged stripe width is $10-15 \mathrm{~nm}$, but there are also domains which are less than $5 \mathrm{~nm}$ in width. The cross section TEM image in Fig. 4(b) shows both vertical domain walls, corresponding to the stripe domain walls, and $45^{\circ}$ domain walls, corresponding to the needle like ending of the stripes. The HRTEM in Fig. 4(c) reveals a sharp interface and no misfit dislocations were visible with these measuring conditions.

Streiffer et al..$^{5}$ predicted the formation of this kind of stripe domains for rhombohedrally distorted ferroelectrics. Because of symmetry reasons, four different pairs of twin domain patterns should occur: for domains with $\{010\}_{c}$ twin boundaries, the patterns $r_{1} / r_{2}$ and $r_{3} / r_{4}$ can exist, for domains with $\{100\}_{c}$ twin boundaries, the patterns $r_{2} / r_{3}$ and $r_{1} / r_{4}$ are possible (Fig. 2 in Ref. 5). In case of our $\mathrm{BiFeO}_{3}$ films under compressive strain on cubic $\mathrm{SrTiO}_{3}$, this mechanism does not seem to be the dominant one, but it is the formation of monodomain islands. The films on the rare earth scandate substrates show one of the four possible twin patterns. The two different in-plane lattice constants of the (110)-cut substrates might reduce the four twin patterns to two, however the existence of only $r_{3} / r_{4}$ twin pattern cannot be explained by this due to symmetry reasons. To rule out the influence of the miscut direction, a $\mathrm{BiFeO}_{3}$ film was grown on a $\mathrm{SmScO}_{3}(110)$ substrate with the miscut in the opposite direction, toward $[0 \overline{10}]_{c}$. Independent from the miscut direction the same structural domain variants $r_{3}$ and $r_{4}$ were formed in the film.

To further investigate the origin of the twin pattern selection and structural variants XRD-RSMs were carried out on all samples. In contrast to PFM and TEM, the XRD measurements allow us to analyze a larger area of the sample, and thus give macroscopic information on the structure of the film. For RSMs performed around $(203)_{c},(\overline{2} 03)_{c},(023)_{c}$, and $(0 \overline{2} 3)_{c}$ reflections, the $\mathrm{BiFeO}_{3}$ peak splits into two peaks, each peak corresponding to two different variants. For the $(203)_{c} /(\overline{2} 03)_{c}$ peaks the x-ray diffraction plane is parallel to the $(010)_{c}$ plane and the variants split up into a $r_{1} / r_{4}$ peak and a $r_{2} / r_{3}$ peak. For 

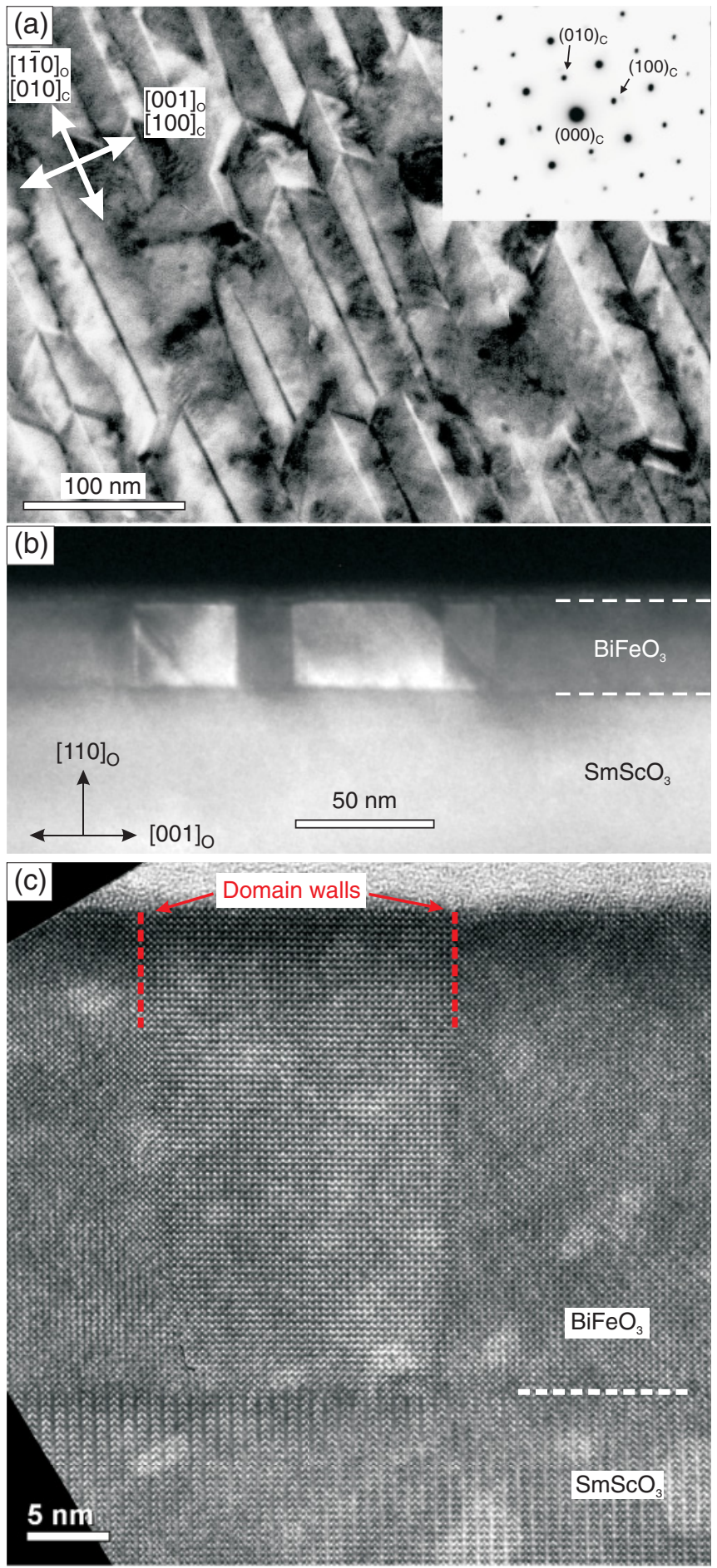

FIG. 4. (Color online) (a) Bright field plan-view TEM image along [110] zone axis of a $26 \mathrm{~nm} \mathrm{BiFeO}_{3}$ grown on $\mathrm{SmScO}_{3}(110)$. (b) Dark field TEM cross section image of $38 \mathrm{~nm}$ thick $\mathrm{BiFeO}_{3}$ film a few degrees out from [1 $1 \overline{1} 0]_{o}$ zone axis to enhance the domain contrast and (c) HRTEM image showing two $109^{\circ}$ domain boundaries in the $\mathrm{BiFeO}_{3}$ film and interface with the $\mathrm{SmScO}_{3}$ substrate.

RSM performed around $(023)_{c}$ or $(0 \overline{2} 3)_{c}$ the $\mathrm{x}$-ray diffraction plane is parallel to $(100)_{c}$ and the two pairs $r_{1} / r_{2}$ and $r_{3} / r_{4}$ can be distinguished. The different RSMs were acquired by rotating the sample in steps of $\phi=90^{\circ}$.
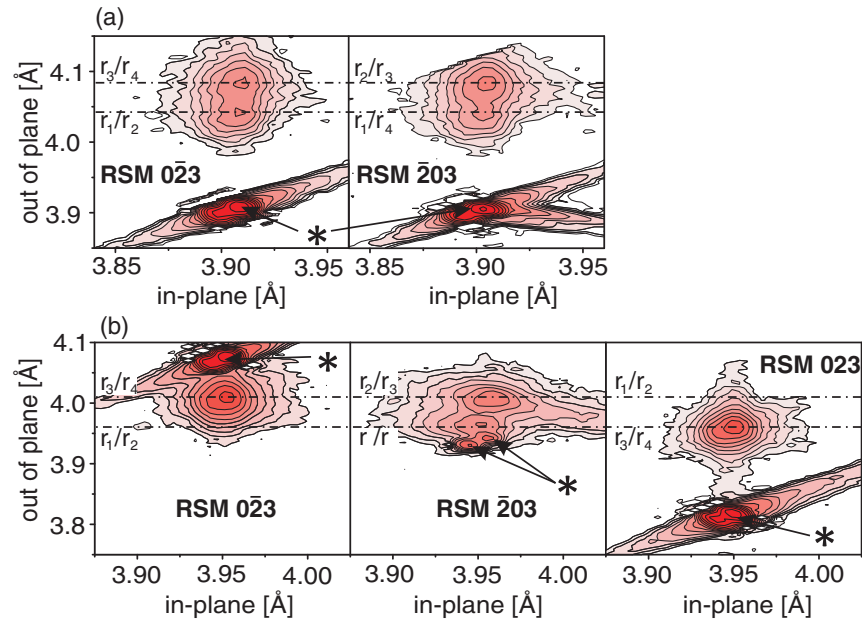

FIG. 5. (Color online) XRD RSMs for a film on (a) $\mathrm{SrTiO}_{3}(001)$ around $(0 \overline{2} 3)_{c}$ and $(\overline{2} 03)_{c}$, as well as for a film on (b) $\operatorname{DyScO}_{3}(110)$ around $(0 \overline{2} 3)_{c},(\overline{2} 03)_{c}$, and $(023)_{c}$. The substrate peaks are labeled with $(*)$. All maps are displayed in logarithmic color code.

As seen in Fig. 5(a) the film on $\mathrm{SrTiO}_{3}$ shows a peak splitting for both RSMs, around $(0 \overline{2} 3)_{c}$ and $(\overline{2} 03)_{c}$. This is in agreement with the PFM result that all four structural domains appear. In contrast, for the films on all of the rare earth scandates, peak splitting was observed only for some RSMs. Figure 5(b) displays the RSMs measured for $\mathrm{BiFeO}_{3}$ on $\mathrm{DyScO}_{3}$ substrate. For $(0 \overline{2} 3)_{c}$ and $(023)_{c}$, only one $\mathrm{BiFeO}_{3}$ peak is visible, whereas for $(\overline{203})_{c}$ and $(203)_{c}$ the peak split is visible $\left[(\overline{2} 03)_{c}\right.$ is identical to $(203)_{c}$, and thus not shown]. In agreement with the PFM measurements [Fig. 3(b)], only the structural variants $r_{3}$ and $r_{4}$ occur in the $\mathrm{BiFeO}_{3}$ film deposited on $\mathrm{DyScO}_{3}$.

Due to the monoclinic structure of the substrate, the substrate peaks appear at different positions for the different RSMs. It can be seen from Fig. 5(b) that the $\mathrm{BiFeO}_{3}$ peak for the RSMs around $(0 \overline{2} 3)_{c}$ and $(023)_{c}$ is close to the $\mathrm{DyScO}_{3}$ peak, meaning that the film tries to adopt the direction of monoclinic distortion of the substrate as depicted in Fig. 6(a). Since the monoclinic distortion of the substrate is along one of the pseudocubic axes and the distortion for $\mathrm{BiFeO}_{3}$ is along one of the diagonals of the pseudocube, there are two equally good possibilities for the $\mathrm{BiFeO}_{3}$ cell to match the substrate distortion, namely by structural variants $r_{3}$ and $r_{4}$. The origin of the selection of the $r_{3} / r_{4}$ stripe patterns seems to be a combination of both: the mechanism of coherent twin boundaries described by Streiffer et al. ${ }^{5}$ and of the preferred direction of distortion due to the monoclinic distorted substrate.

Furthermore, the $\mathrm{BiFeO}_{3}$ films under tensile strain on $\mathrm{GdScO}_{3}$ and $\mathrm{SmScO}_{3}$ show a thickness dependent peak splitting in the RSM around (203) . The RSMs for films on $\mathrm{SmScO}_{3}$ with thicknesses of 13,26 , and $38 \mathrm{~nm}$ are depicted in Fig. 7. The $\mathrm{BiFeO}_{3}$ films on $\mathrm{GdScO}_{3}$ display the same behavior, so the data is not shown here. The RSM data is shown in two representations, first $(\Theta-\omega)$ versus $2 \Theta$ and second transformed into lattice parameters $a$ and $c$. In the first representation $(\Theta-\omega)$ gives the tilt of the planes under investigation with respect to the substrate surface $(001)_{c}$, 
(a)

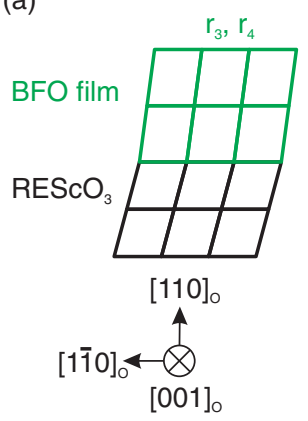

(b)

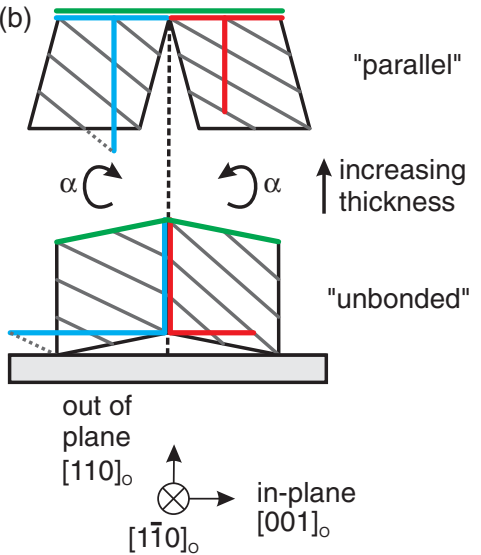

$11 \overline{1} 0]$

FIG. 6. (Color online) (a) An epitaxially strained $\mathrm{BiFeO}_{3}$ film prefers a structural variant which adapts to the monoclinic distortion of the rare earth substrates. (b) Schematics of the rotation of the $\mathrm{BiFeO}_{3}$ unit cell with thickness. (203) $)_{c}$ planes (gray lines) and extracted in-plane and out-of-plane lattice constants shown for both structural variants (red and blue lines, respectively), leading to a transition from a vertical to a horizontal peak splitting in RSMs. Green line shows orientation of the $(004)_{c}$ plane in both cases.

whereas from the $2 \Theta$ value the plane spacing can be deduced. In the other case, from the calculated lattice parameters $a$ and $c$ the plane distances parallel to the sample surface and perpendicular to the sample surface can be derived, respectively. As seen in Figs. 7(a)-7(c), for all thicknesses the (203) plane spacing stays constant, but the planes seem to have different tilt angles with respect to the substrate surface. Starting with the $13 \mathrm{~nm}$ thick film, the additional layers from the two structural variants rotate in opposite directions. For the $38 \mathrm{~nm}$ thick sample, the planes form reflections with maximal intensity at different positions with respect to the thinner films. The different orientation of the first layers is, however, still visible as a tail. The $26 \mathrm{~nm}$ thick $\mathrm{BiFeO}_{3}$ film is close to the transition with no pronounced peak reflection at none of the two positions [Figs. 7(b) and 7(e)]. On the other hand, when converted into lattice constants, with increasing film thickness there is a transition from a horizontal splitting into a vertical splitting. This means that the first $\mathrm{BiFeO}_{3}$ layers for both structural variants have the same $(203)_{c}$ plane spacing projected on the out of plane direction, in contrast to the layers thicker than $\approx 26 \mathrm{~nm}$, which have the same $(203)_{c}$ plane spacing projected on the in-plane direction. A possible explanation is a rotation of the unit cell as depicted in Fig. 6(b). Starting from an unbonded configuration with the substrate surface, the unit cell rotates with increasing thickness to a parallel alignment with the substrate surface. For a further information RSMs around (004) $c$ were performed. The unbonded case should show a peak splitting due to the two different plane orientations, whereas in the parallel case only one peak is expected [Fig. 6(b)]. As can be seen in Figs. 7(g) and 7(h) the measurements are in agreement with this consideration. A further evidence is the amount of rotation $\alpha$ of $0.3^{\circ}$ to $0.4^{\circ}$ which exactly match with the rhombohedral distortion of $\mathrm{BiFeO}_{3}$ projected onto the $(010)_{c}$ plane, which is seen by these XRD measurements. This rotation might be one possible way to relax part of the strain.
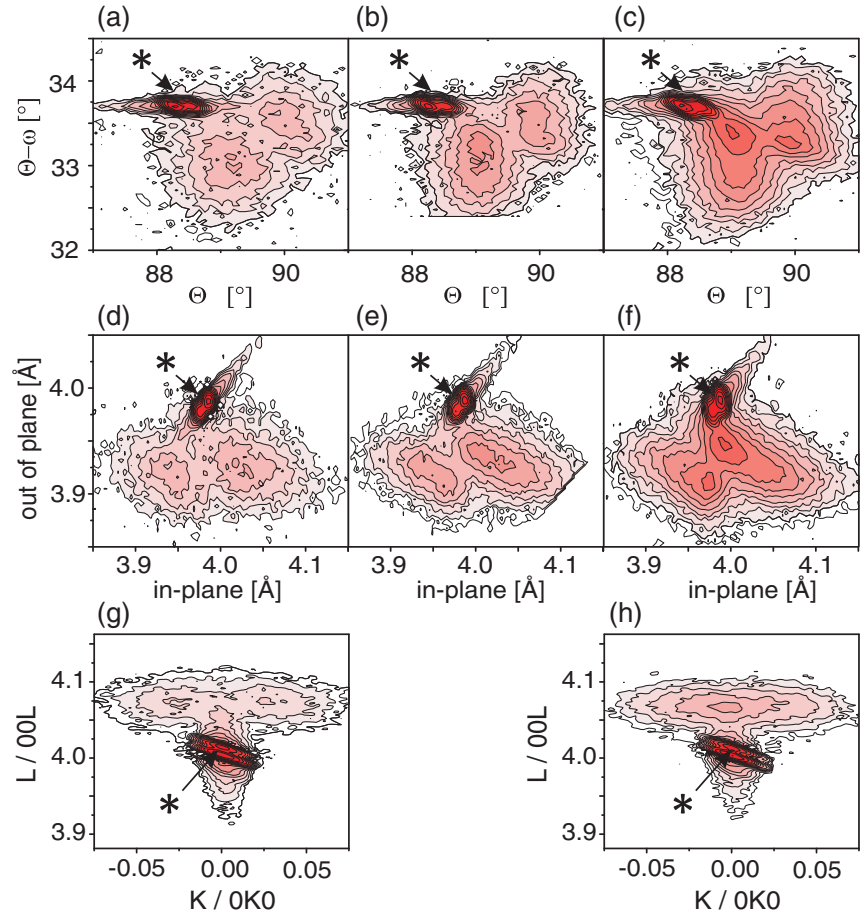

FIG. 7. (Color online) XRD-RSMs around (203) $)_{c}$ of $\mathrm{BiFeO}_{3}$ films grown on $\mathrm{SmScO}_{3}(110)$. Films with three different thicknesses were measured: (a) and (d) $13 \mathrm{~nm}$, (b) and (e) $26 \mathrm{~nm}$, (c) and (f) $38 \mathrm{~nm}$, and the data are shown in two ways, $\Theta-\omega$ vs $2 \Theta$ (a)-(c) and transformed into lattice parameters (d)-(f). XRD-RSMs around (004) for (g) $13 \mathrm{~nm}$ and (h) $38 \mathrm{~nm}$ film on $\mathrm{SmScO}_{3}(110)$. The $\mathrm{K}$ and $\mathrm{L}$ axes are the Miller indices in pseudocubic notation for the $\mathrm{SmScO}_{3}$ substrate and are parallel to the in-plane and out-of-plane direction of the sample surface. The substrate peaks are labeled with (*). All maps are displayed in logarithmic color code.

\section{B. $\mathrm{BiFeO}_{3}$ films grown with $\mathrm{SrRuO}_{3}$ bottom electrode}

The $\mathrm{SrRuO}_{3}$ bottom electrode exerts important influence on the $\mathrm{BiFeO}_{3}$ film growth and properties due to its different termination, electrical properties, structure, and film quality. For $\mathrm{SrTiO}_{3}$ and $\mathrm{DyScO}_{3}$ substrates, high quality $\mathrm{SrRuO}_{3}$ films were achieved. XRD measurements reveal fully strained films (Fig. 10) and AFM measurements show that the terrace structure of the substrate is maintained (not shown). However, for $\mathrm{GdScO}_{3}$ and $\mathrm{SmScO}_{3}$ the lattice mismatch between $\mathrm{SrRuO}_{3}$ and the substrate is so large that even 5 to $10 \mathrm{~nm}$ thick $\mathrm{SrRuO}_{3}$ films relax partly. From XRD measurements, it can be inferred that the strain acting on $\mathrm{BiFeO}_{3}$ is actually $-0.1 \%$ and $+0.5 \%$ for $\mathrm{GdScO}_{3}$ and $\mathrm{SmScO}_{3}$ substrates, respectively. This means that even though the pseudocubic lattice constant of $\mathrm{GdScO}_{3}$ is slightly bigger than the one of $\mathrm{BiFeO}_{3}$, the $\mathrm{BiFeO}_{3}$ film on $\mathrm{GdScO}_{3}$ substrate with $\mathrm{SrRuO}_{3}$ bottom electrode is compressively strained. In case of the $\mathrm{GdScO}_{3}$ substrate, the $\mathrm{SrRuO}_{3}$ morphology exhibits terraces on which small islands were formed, whereas for $\mathrm{SmScO}_{3}$ substrates the $\mathrm{SrRuO}_{3}$ showed mainly an island growth mode. Due to the bad $\mathrm{SrRuO}_{3}$ film quality on $\mathrm{GdScO}_{3}$ and $\mathrm{SmScO}_{3}$, the growth conditions of $\mathrm{BiFeO}_{3}$ differ in comparison to $\mathrm{SrTiO}_{3}$ and $\mathrm{DyScO}_{3}$ substrates on which high quality $\mathrm{SrRuO}_{3}$ bottom electrodes were grown. Moreover, it is likely that the electronic properties of the $\mathrm{SrRuO}_{3}$ layers grown on 

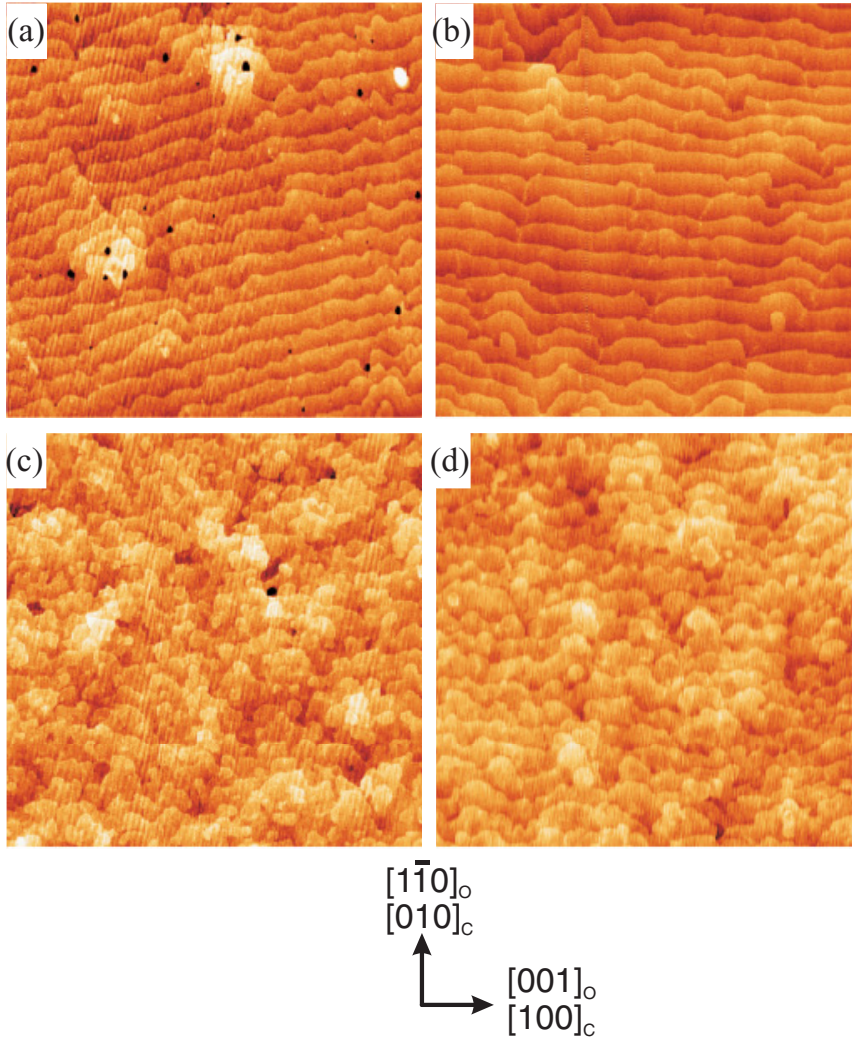

FIG. 8. (Color online) AFM images of the surface morphology of $30 \mathrm{~nm}$ thick $\mathrm{BiFeO}_{3}$ films grown on (a) $\mathrm{SrTiO}_{3}(001)$, (b) $\mathrm{DyScO}_{3}(110)$, (c) $\mathrm{GdScO}_{3}(110)$, and (d) $\mathrm{SmScO}_{3}(110)$, with $\mathrm{SrRuO}_{3}$ bottom electrode. All images are $4 \times 4 \mu \mathrm{m}^{2}$.

$\mathrm{GdScO}_{3}$ and $\mathrm{SmScO}_{3}$ are significantly different than for the $\mathrm{SrRuO}_{3}$ deposited on $\mathrm{SrTiO}_{3}$ and $\mathrm{DyScO}_{3} .{ }^{25}$ Figure 8 shows the morphology of the $\mathrm{BiFeO}_{3}$ films on all four substrates. All films exhibit one unit cell high terraces, although more disordered for films on $\mathrm{GdScO}_{3}$ and $\mathrm{SmScO}_{3}$.

To investigate the domain configuration PFM measurements were performed on all films. The VPFM images reveal that all films have an uniform polarization pointing downward, probably due to the electrical properties of $\mathrm{SrRuO}_{3},{ }^{9}$ and are not shown here. In the case of $\mathrm{SrTiO}_{3}$ and $\mathrm{DyScO}_{3}$ substrates, the LPFM images depend again strongly on the cantilever orientation, as can be seen in Figs. 9(a) and 9(b). While scanning with the cantilever aligned perpendicular to the terraces the films show an evenly distributed domain distribution, with different domain sizes and shapes. On the other hand, by scanning with the cantilever aligned along the terraces, a preferred contrast occurs in the LPFM images. Combining VPFM and both LPFM images one can conclude that for $\mathrm{SrTiO}_{3}$ and $\mathrm{DyScO}_{3}$ substrates only the structural variants $r_{3}$ and $r_{4}$ occur, with only the two polarization directions $P_{3}^{-}$and $P_{4}^{-}$[Fig. 9(e)]. The domain patterns in the LPFM images for films on $\mathrm{GdScO}_{3}$ and $\mathrm{SmScO}_{3}$ substrates are very similar and show almost no preferred domain variants for both cantilever orientation [Figs. 9(c) and 9(d)]. Therefore all structural variants with the polarization directions $P_{1}^{-}, P_{2}^{-}, P_{3}^{-}$, and $P_{4}^{-}$occur. The appearance of all structural variants is in agreement with the island formation during the growth of the $\mathrm{SrRuO}_{3}$ layer which annihilates the possible effects of the substrate asymmetry.

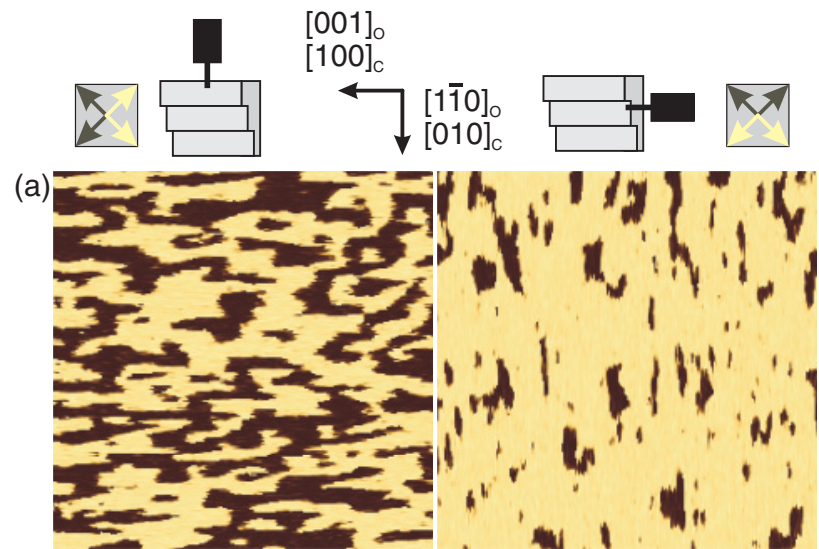

(b)
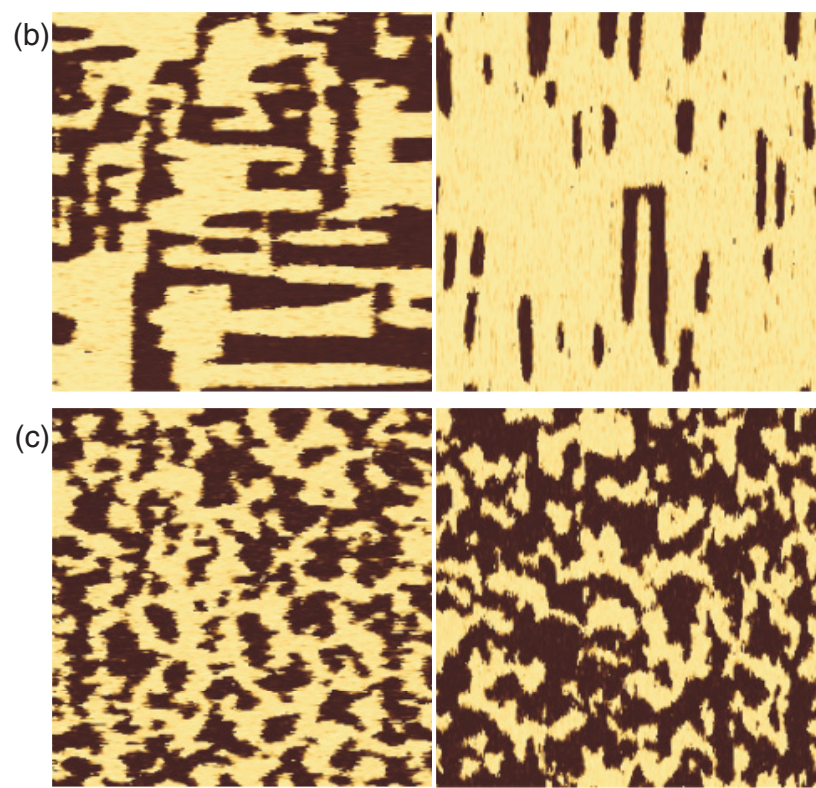

(d)

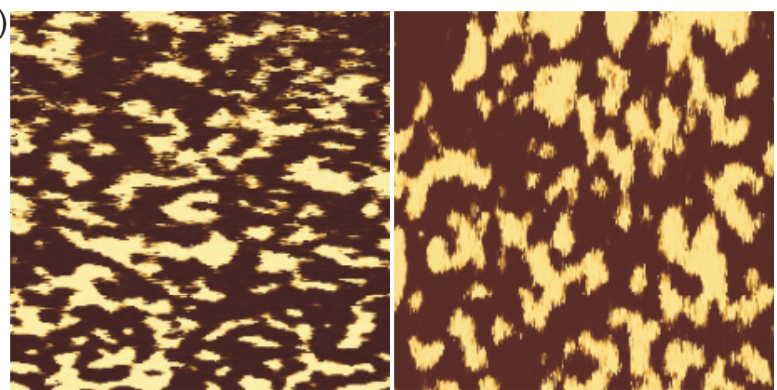

(e)

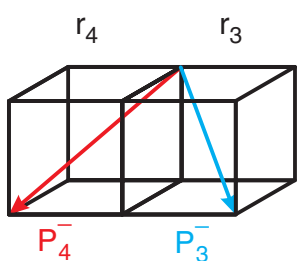

(f)

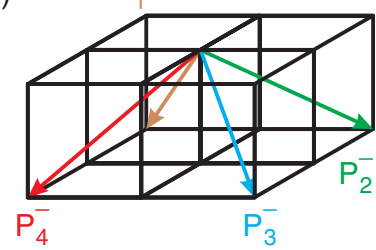

FIG. 9. (Color online) LPFM images of BFO films with $\mathrm{SrRuO}_{3}$ bottom electrode on (a) $\mathrm{SrTiO}_{3}(100)$, (b) $\mathrm{DyScO}_{3}(110)$, (c) $\mathrm{GdScO}_{3}(110)$, and (d) $\mathrm{SmScO}_{3}(110)$. Samples were measured with the cantilever oriented along $[010]_{c}$ (left image) and $[100]_{c}$ (right image). The insets in the LPFM images show the two possible polarization directions for both contrasts. All images are $3 \times 3 \mu \mathrm{m}^{2}$. Preferred directions of polarization are indicated for $\mathrm{BFO}$ films on (e) $\mathrm{SrTiO}_{3}$ and $\mathrm{DyScO}_{3}$ and for (f) $\mathrm{GdScO}_{3}$ and $\mathrm{SmScO}_{3}$. 
(a)

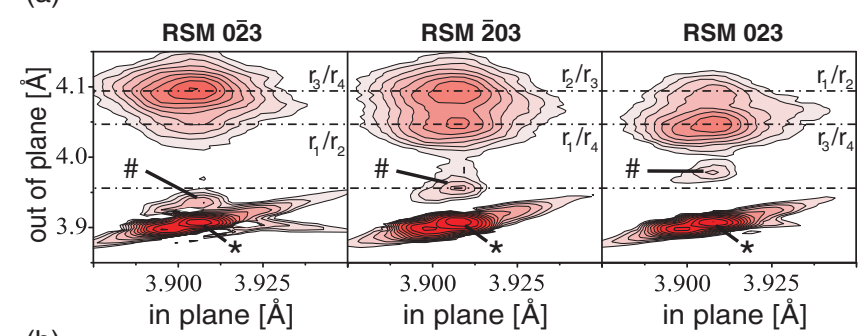

(b)

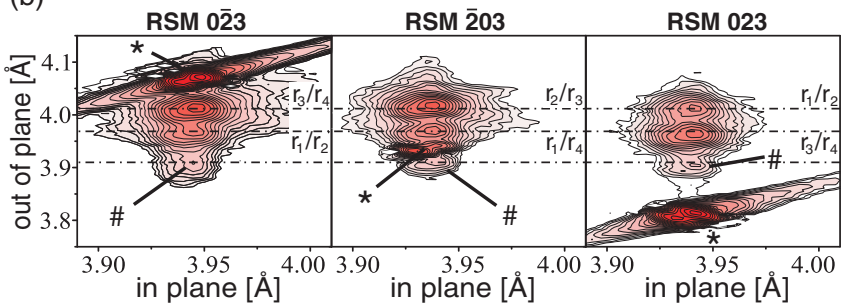

FIG. 10. (Color online) XRD-RSMs around (203), (023), and (203) for a film on (a) $\mathrm{SrTiO}_{3}$ and (b) $\mathrm{DyScO}_{3}$ substrates with $\mathrm{SrRuO}_{3}$ bottom electrode. Substrate peaks and $\mathrm{SrRuO}_{3}$ peaks are labeled with $(*)$ and $(\#)$, respectively.

In order to investigate the origin of the preferred directions observed for $\mathrm{BiFeO}_{3}$ in case of $\mathrm{SrTiO}_{3}$ and $\mathrm{DyScO}_{3}$ substrates, RSMs around $(\overline{2} 03)_{c},(023)_{c}$ and $(203)_{c}$ were measured on those films. Figure 10(a) shows the result for the $\mathrm{SrTiO}_{3}$ case. The peaks for $\mathrm{SrRuO}_{3}$ have different out-of-plane parameters in the different RSMs, indicating that the $\mathrm{SrRuO}_{3}$ layer is monodomain and that it kept its orthorhombic unit cell with a monoclinic distorted pseudocube. ${ }^{26}$ The crystallographic orientation of $\mathrm{SrRuO}_{3}$ is determined by the miscut direction. As sketched in Fig. 11(a) the occurring distortions in the $\mathrm{BiFeO}_{3}$ films are opposed to the monoclinic ones of $\mathrm{SrRuO}_{3}$. The origin of the domain suppression are the substrate terraces, which break the cubic substrate symmetry. The $\mathrm{BiFeO}_{3}$ films grown directly on the $\mathrm{SrTiO}_{3}$ substrate do not show this domain selection. Those films grow in a different mode as well, as they do not exhibit terraces anymore. Therefore, the electrical properties and surface chemistry of the $\mathrm{SrRuO}_{3}$ layer appear to be necessary for the preferred domain variants of the $\mathrm{BiFeO}_{3}$ on $\mathrm{SrRuO}_{3} / \mathrm{SrTiO}_{3}(001)$.

The RSMs for the film on $\mathrm{DyScO}_{3}$ are shown in Fig. 10(b). The $\mathrm{SrRuO}_{3}$ grows on $\mathrm{DyScO}_{3}(110)$ a tetragonal structure, as reported by Vailionis et al. ${ }^{27}$ The distortions of $\mathrm{BiFeO}_{3}$ are aligned in the same direction as the distortion of the substrate [Fig. 11(b)], similar to the case without the $\mathrm{SrRuO}_{3}$ bottom electrode. However, with respect to the terrace alignment these are the same structural variants as on $\mathrm{SrTiO}_{3}$. To determine the origin of the domain selection in case of $\mathrm{DyScO}_{3}$ substrates, whether it is the terrace alignment or the substrate distortion, films were grown on substrates with opposite miscut directions, as depicted in Figs. 11(b) and 11(c). PFM and XRD measurements reveal (not shown) that the preferred domain variants change to $r_{1}$ and $r_{2}$ when changing the offcut direction. This proves that the terrace alignment is the driving mechanism for domain suppression.

Macroscopic ferroelectric polarization measurements were performed to study the influence of epitaxial strain and domain variants on the magnitude of the switchable polarization. (a)

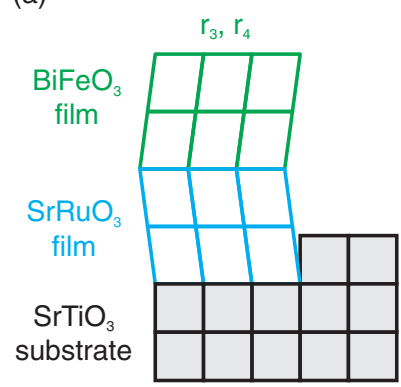

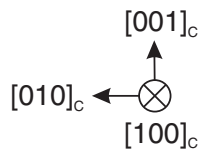

(b)

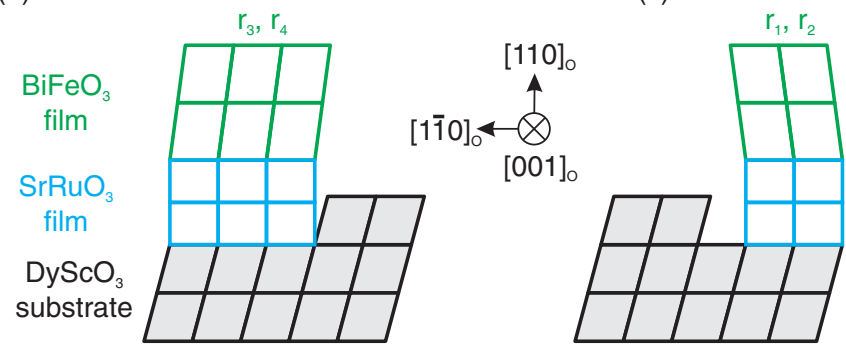

FIG. 11. (Color online) Schematics of preferred domain configuration for $\mathrm{BiFeO}_{3}$ films with $\mathrm{SrRuO}_{3}$ bottom electrode on $\mathrm{SrTiO}_{3}$ (a) and $\mathrm{DyScO}_{3}$ with two different miscut direction (b) and (c).

Amorphous $\mathrm{SrRuO}_{3}$ top electrodes covered with platinum $\left(60 \times 60 \mu \mathrm{m}^{2}\right.$ in size $)$ were deposited on the $30 \mathrm{~nm}$ thick $\mathrm{BiFeO}_{3}$ films. The voltage was applied to the top electrode. Figures 12(a) and 12(b) show the ferroelectric polarization and switching currents of $\mathrm{BiFeO}_{3}$ films on $\mathrm{SrRuO}_{3}$-covered $\mathrm{SrTiO}_{3}, \mathrm{DyScO}_{3}$, and $\mathrm{GdScO}_{3}$ substrates. In agreement with other reports, ${ }^{28,29}$ there is a slight decrease of switchable polarization for $\mathrm{BiFeO}_{3}$ films under tensile strain. As mentioned already above, the film on $\mathrm{GdScO}_{3}$ is not fully strained.

For the film on $\mathrm{SmScO}_{3}$, smaller electrodes had to be chosen because of existing leakage paths for bigger ones. Hence, $12 \times$ $12 \mu \mathrm{m}^{2}$ amorphous platinum electrodes were deposited on top of the sample. The drawback of the small electrodes lies in the big relative error of more than $25 \%$ for the electrode area determination. Therefore, the data from the film on $\mathrm{SmScO}_{3}$ cannot be compared reliably with the other films. It can be seen however, that the ferroelectric polarization is of the same order of magnitude.

Although the different films studied here have different number of structural variants, the spontaneous polarization has the same order of magnitude. In contrast to what was reported for $\mathrm{BiFeO}_{3}$ on low miscut $\mathrm{SrTiO}_{3}(001)$ substrates with four structural variants we see no major difference in the magnitude of switchable polarization. ${ }^{7}$ There seem to be no pinned domain walls in our films with four structural variants.

All films have a strong imprint of about $-550 \mathrm{kV} / \mathrm{cm}$. To exclude the possibly bad interface with the amorphous $\mathrm{SrRuO}_{3}$ top electrode as an origin of this imprint, a $\mathrm{BiFeO}_{3}$ film with $\mathrm{SrRuO}_{3}$ bottom electrode and in situ epitaxial top electrode was grown. The in situ epitaxial top electrodes had to be patterned in the top film afterwards by an etching procedure. Because $\mathrm{SrRuO}_{3}$ is very inert to acids, $\mathrm{La}_{0.7} \mathrm{Sr}_{0.3} \mathrm{MnO}_{3}$ (LSMO) was chosen as epitaxial top electrode, with the drawback of an electrically asymmetric $\mathrm{LSMO} / \mathrm{BiFeO}_{3} / \mathrm{SrRuO}_{3}$ 
(a)

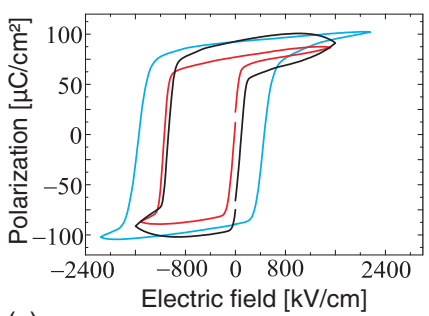

(c)
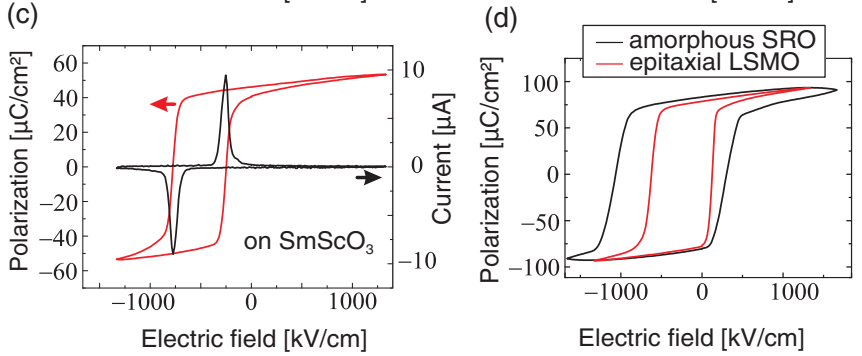

FIG. 12. (Color online) Ferroelectric polarization loop (a) and its corresponding current (b) for films on $\mathrm{SrRuO}_{3}$-covered $\mathrm{SrTiO}_{3}$, $\mathrm{DyScO}_{3}$, and $\mathrm{GdScO}_{3}$ measured at $100 \mathrm{~K}$ and $1 \mathrm{kHz}$ with amorphous $\mathrm{SrRuO}_{3}$ top electrodes $\left(60 \times 60 \mu \mathrm{m}^{2}\right)$. (c) Ferroelectric polarization and corresponding current for film on $\mathrm{SmScO}_{3}$, measured at $100 \mathrm{~K}$ and $1 \mathrm{kHz}$ with amorphous platinum top electrodes $\left(12 \times 12 \mu \mathrm{m}^{2}\right)$. (d) Ferroelectric polarization loop for films on $\mathrm{DyScO}_{3}$ substrate with $60 \times 60 \mu \mathrm{m}^{2}$ amorphous $\mathrm{SrRuO}_{3}$ and epitaxial $\mathrm{La}_{0.7} \mathrm{Sr}_{0.3} \mathrm{MnO}_{3}$ top electrodes, measured at $10 \mathrm{~K}$ and $1 \mathrm{kHz}$.

system. Platinum capacitors of $60 \times 60 \mu \mathrm{m}^{2}$ in size were deposited on the epitaxial LSMO top electrode and the remaining LSMO layer was etched. As seen in Fig. 12(d) the film with epitaxial LSMO has slightly lower imprint, but it is still very pronounced. The coercive field is, however, distinctly reduced in case of epitaxial electrodes. Hence, the quality of the interface with the electrodes seems to be an important parameter for the coercive field.

The imprint might be connected to the polarization pointing preferentially toward the $\mathrm{SrRuO}_{3}$ bottom electrode. In contrast, the films grown on the insulating substrate had a preferred direction upward, especially for $\mathrm{BiFeO}_{3}$ grown on $\mathrm{SrTiO}_{3}$ [Fig. 3(a)]. A uniform polarization pointing toward the $\mathrm{SrRuO}_{3}$ bottom electrode of a $\mathrm{La}: \mathrm{BiFeO}_{3}$ film has already been reported and could be correlated to the surface carrier density of $\mathrm{SrRuO}_{3}$, which allows a full screening of the polarization at the interface. ${ }^{9}$ There are many reports for several material systems in literature, showing that there exists a preferred direction of polarization, which depends on the type of interface. For example, the direction of polarization for a $30 \mathrm{~nm}$ thick $\mathrm{Pb}\left(\mathrm{Zi}_{0.2} \mathrm{Ti}_{0.8}\right) \mathrm{O}_{3}$ film on LSMO bottom electrode points downward with an imprint of $-1.2 \mathrm{~V}$, whereas for $\mathrm{SrRuO}_{3}$ bottom electrode the polarization is pointing upward. ${ }^{30}$ In $\mathrm{PbTiO}_{3}$ films grown on $\mathrm{SrRuO}_{3} / \mathrm{SrTiO}_{3}$ a transition from a polarization pointing downward for films thinner than $20 \mathrm{~nm}$ to a polarization pointing upward for thicker films occurs. ${ }^{31}$ In addition, for $\mathrm{BiFeO}_{3}$ on LSMO electrode it has been reported that there exists a strong pinning of the polarization at the interface, due to an induced polarization in the LSMO layer. ${ }^{32}$ Hence, among others, the role of the interface with the bottom electrode seems to be very important in imposing a preferential orientation of epitaxial ferroelectric films. Some analytical tools might help to understand this behavior, for example, soft x-ray scattering investigations of the $\mathrm{Pb}(\mathrm{Zr}, \mathrm{Ti}) \mathrm{O}_{3} / \mathrm{LSMO}$ system revealed that the $\mathrm{Ti}^{4+}$ ions at the interface have a different $3 d$-orbital character depending on the direction of polarization. ${ }^{33}$ Therefore, a preferred direction of polarization might be a way to minimize the interfacial energy.

\section{CONCLUSIONS}

The domain variants formed in epitaxial $\mathrm{BiFeO}_{3}$ films grown on single crystal substrates depend strongly on several factors: the growth mode of the $\mathrm{BiFeO}_{3}$ film, the type of interface, the substrate symmetry, and the substrate miscut direction.

$\mathrm{BiFeO}_{3}$ films grown directly on cubic $\mathrm{SrTiO}_{3}$ substrates exhibit all four structural domain variants, whereas for films grown on the orthorhombic rare earth scandates two structural domain variants are suppressed, which is a result of the monoclinic symmetry of these substrates when cut in (110) direction. In addition, stripe domain patterns were formed regardless of compressive and tensile stresses. For tensile strain, as it is the case of $\mathrm{BiFeO}_{3}$ grown on $\mathrm{GdScO}_{3}(110)$ and $\mathrm{SmScO}_{3}(110)$, the stripes have widths smaller than $10 \mathrm{~nm}$ for $26 \mathrm{~nm}$ thick films, and a rotation of the $\mathrm{BiFeO}_{3}$ unit cell occurs within the first $20-30 \mathrm{~nm}$.

Coherently strained $\mathrm{BiFeO}_{3}$ films grown on $\mathrm{SrTiO}_{3}$ and $\mathrm{DyScO}_{3}$ substrates with a step flow grown $\mathrm{SrRuO}_{3}$ bottom electrode maintained the terrace morphology of the $0.15^{\circ}$ miscut substrate, which, in contrast to the films without $\mathrm{SrRuO}_{3}$, plays an important role in the structural domain selection. Films on $\mathrm{GdScO}_{3}$ and $\mathrm{SmScO}_{3}$ with $\mathrm{SrRuO}_{3}$ electrode are partly relaxed and do not show any structural domain suppression. This might be due to the bad $\mathrm{SrRuO}_{3}$ surface morphology, because step-flow growth could not be achieved anymore. All films show very similar values for the switchable polarization, independently of the number of structural variants within the film and of strain state.

\section{ACKNOWLEDGMENTS}

F. J. would like to thank Dr. Wei Peng for fruitful discussions and help with the XRD measurements. Financial support by DFG through SFB 762 is gratefully acknowledged.

\footnotetext{
*fjohann@mpi-halle.de

${ }^{1}$ N. A. Spaldin and M. Fiebig, Science 309, 391 (2005).

${ }^{2}$ W. Eerenstein, N. D. Mathur, and J. F. Scott, Nature (London) 442, 759 (2006).

${ }^{3}$ G. Catalan and J. F. Scott, Adv. Mater. 21, 2463 (2009).
}

${ }^{4}$ F. Kubel and H. Schmid, Acta Crystallogr. Sect. B 46, 698 (1990).

${ }^{5}$ S. K. Streiffer, C. B. Parker, A. E. Romanov, M. J. Lefevre, L. Zhao, J. S. Speck, W. Pompe, C. M. Foster, and G. R. Bai, J. Appl. Phys. 83, 2742 (1998). 
${ }^{6}$ T. Zhao, A. Scholl, F. Zavaliche, K. Lee, M. Barry, A. Doran, M. P. Cruz, Y. H. Chu, C. Ederer, N. A. Spaldin, R. R. Das, D. M. Kim, S. H. Baek, C. B. Eom, and R. Ramesh, Nat. Mater. 5, 823 (2006).

${ }^{7}$ J. W. Park, S. H. Baek, P. Wu, B. Winchester, C. T. Nelson, X. Q. Pan, L. Q. Chen, T. Tybell, and C. B. Eom, Appl. Phys. Lett. 97, 212904 (2010).

${ }^{8}$ H. W. Jang, D. Ortiz, S. H. Baek, C. M. Folkman, R. R. Das, P. Shafer, Y. Chen, C. T. Nelson, X. Pan, R. Ramesh, and C. B. Eom, Adv. Mater. 21, 817 (2009).

${ }^{9}$ Y. H. Chu, Q. He, C. H. Yang, P. Yu, L. W. Martin, P. Shafer, and R. Ramesh, Nano Lett. 9, 1726 (2009).

${ }^{10}$ S. Geller and E. A. Wood, Acta Crystallogr. 9, 563 (1956).

${ }^{11}$ R. Uecker, B. Velickov, D. Klimm, R. Bertram, M. Bernhagen, M. Rabe, M. Albrecht, R. Fornari, and D. G. Schlom, J. Cryst. Growth 310, 2649 (2008).

${ }^{12}$ Y.-H. Chu, Q. Zhan, L. Martin, M. Cruz, P.-L. Yang, G. Pabst, F. Zavaliche, S.-Y. Yang, J.-X. Zhang, L.-Q. Chen, D. Schlom, I.-N. Lin, T.-B. Wu, and R. Ramesh, Adv. Mater. 18, 2307 (2006).

${ }^{13}$ C. M. Folkman, S. H. Baek, H. W. Jang, C. B. Eom, C. T. Nelson, X. Q. Pan, Y. L. Li, L. Q. Chen, A. Kumar, V. Gopalan, and S. K. Streiffer, Appl. Phys. Lett. 94, 251911 (2009).

${ }^{14}$ C. T. Nelson, B. Winchester, Y. Zhang, S. J. Kim, A. Melville, C. Adamo, C. M. Folkman, S. H. Baek, C. B. Eom, D. G. Schlom, L. Q. Chen, and X. Q. Pan, Nano Lett. 11, 828 (2011).

${ }^{15}$ B. Dupé, S. Prosandeev, G. Geneste, B. Dkhil, and L. Bellaiche, Phys. Rev. Lett. 106, 237601 (2011).

${ }^{16}$ G. Koster, B. L. Kropman, G. J. H. M. Rijnders, D. H. A. Blank, and H. Rogalla, Appl. Phys. Lett. 73, 2920 (1998).

${ }^{17}$ Y.-H. Chu, T. Zhao, M. P. Cruz, Q. Zhan, P. L. Yang, L. W. Martin, M. Huijben, C. H. Yang, F. Zavaliche, H. Zheng, and R. Ramesh, Appl. Phys. Lett. 90, 252906 (2007).

${ }^{18}$ F. Zavaliche, S. Y. Yang, T. Zhao, Y. H. Chu, M. P. Cruz, C. B. Eom, and R. Ramesh, Phase Transitions 79, 991 (2006).

${ }^{19}$ D. B. Williams and C. B. Carter, Transmission Electron Microscopy (Springer, New York, 1996).
${ }^{20}$ K. Saito, A. Ulyanenkov, V. Grossmann, H. Ress, L. Bruegemann, H. Ohta, T. Kurosawa, S. Ueki, and H. Funakubo, Jpn. J. Appl. Phys. 45, 7311 (2006).

${ }^{21}$ C. J. M. Daumont, S. Farokhipoor, A. Ferri, J. C. Wojdeł, J. Íñiguez, B. J. Kooi, and B. Noheda, Phys. Rev. B 81, 144115 (2010).

${ }^{22}$ J. E. Kleibeuker, G. Koster, W. Siemons, D. Dubbink, B. Kuiper, J. L. Blok, C.-H. Yang, J. Ravichandran, R. Ramesh, J. E. ten Elshof, D. H. A. Blank, and G. Rijnders, Adv. Funct. Mater. 20, 3490 (2010).

${ }^{23}$ G. Rijnders, D. H. A. Blank, J. Choi, and C.-B. Eom, Appl. Phys. Lett. 84, 505 (2004).

${ }^{24}$ T. Jungk, A. Hoffmann, and E. Soergel, New J. Phys. 10, 013019 (2008).

${ }^{25}$ A. T. Zayak, X. Huang, J. B. Neaton, and K. M. Rabe, Phys. Rev. B 74, 094104 (2006).

${ }^{26}$ Q. Gan, R. A. Rao, and C. B. Eom, Appl. Phys. Lett. 70, 1962 (1997).

${ }^{27}$ A. Vailionis, W. Siemons, and G. Koster, Appl. Phys. Lett. 93, 051909 (2008)

${ }^{28}$ H. W. Jang, S. H. Baek, D. Ortiz, C. M. Folkman, R. R. Das, Y. H. Chu, P. Shafer, J. X. Zhang, S. Choudhury, V. Vaithyanathan, Y. B. Chen, D. A. Felker, M. D. Biegalski, M. S. Rzchowski, X. Q. Pan, D. G. Schlom, L. Q. Chen, R. Ramesh, and C. B. Eom, Phys. Rev. Lett. 101, 107602 (2008).

${ }^{29}$ M. D. Biegalski, D. H. Kim, S. Choudhury, L. Q. Chen, H. M. Christen, and K. Dörr, Appl. Phys. Lett. 98, 142902 (2011).

${ }^{30}$ P. Maksymovych, S. Jesse, P. Yu, R. Ramesh, A. P. Baddorf, and S. V. Kalinin, Science 324, 1421 (2009).

${ }^{31} \emptyset$. Dahl, J. K. Grepstad, and T. Tybell, J. Appl. Phys. 106, 084104 (2009).

${ }^{32}$ H. J. Chang, S. V. Kalinin, A. N. Morozovska, M. Huijben, Y.-H. Chu, P. Yu, R. Ramesh, E. A. Eliseev, G. S. Svechnikov, S. J. Pennycook, and A. Y. Borisevich, Adv. Mater. 23, 2474 (2011).

${ }^{33}$ E. Arenholz, G. van der Laan, A. Fraile-Rodríguez, P. Yu, Q. He, and R. Ramesh, Phys. Rev. B 82, 140103 (2010). 